\title{
Photonic crystal fiber based antibody detection
}

Duval, A; Lhoutellier, M; Jensen, J B; Hoiby, P E; Missier, V; Pedersen, L H; Hansen, Theis Peter; Bjarklev, Anders Overgaard; Bang, Ole

\section{Published in:}

Proceedings of IEEE Sensors, 2004.

Link to article, DOI:

10.1109/ICSENS.2004.1426399

Publication date:

2004

Document Version

Publisher's PDF, also known as Version of record

Link back to DTU Orbit

Citation (APA):

Duval, A., Lhoutellier, M., Jensen, J. B., Hoiby, P. E., Missier, V., Pedersen, L. H., Hansen, T. P., Bjarklev, A. O., \& Bang, O. (2004). Photonic crystal fiber based antibody detection. In Proceedings of IEEE Sensors, 2004. (Vol. 3, pp. 1222-1225). [W1L-D.5] IEEE. I E E E Sensors. Proceedings https://doi.org/10.1109/ICSENS.2004.1426399

\section{General rights}

Copyright and moral rights for the publications made accessible in the public portal are retained by the authors and/or other copyright owners and it is a condition of accessing publications that users recognise and abide by the legal requirements associated with these rights.

- Users may download and print one copy of any publication from the public portal for the purpose of private study or research.

- You may not further distribute the material or use it for any profit-making activity or commercial gain

- You may freely distribute the URL identifying the publication in the public portal 


\title{
Photonic Crystal Fiber Based Antibody Detection
}

\author{
A. Duval ${ }^{1}$, M. Lhoutellier ${ }^{1}$, J.B. Jensen ${ }^{1}$, P.E. Hoiby ${ }^{2}$, V. Missier ${ }^{1}$, L.H. Pedersen ${ }^{2}$, \\ T.P. Hansen ${ }^{1,3}$, A. Bjarklev ${ }^{1}$, and $O{ }^{2}$. Bang ${ }^{1}$ \\ ${ }^{1}$ Research Center COM, DTU Building 345v, Kgs. Lyngby, Denmark, jbj@com.dtu.dk \\ ${ }^{2}$ Bioneer A/S, Kogle Alle 2, DK-2970, Hørsholm, Denmark \\ ${ }^{3}$ Crystal Fibre A/S, Blokken 84, DK-3460 Birkerød, Denmark
}

\begin{abstract}
An original approach for detecting labeled antibodies based on strong penetration photonic crystal fibers is introduced. The target antibody is immobilized inside the air-holes of a photonic crystal fiber and the detection is realized by the means of evanescent-wave fluorescence spectroscopy and the use of a transversal illumination setup.
\end{abstract}

\section{Keywords}

Biosensor, Photonic Crystal Fiber, Cy3, Antibody detection, Fluorescence

\section{INTRODUCTION}

We present here what we believe to be the first implementation of a Photonic Crystal Fiber (PCF) [1] based sensor for selective antibody detection using evanescent-wave fluorescence spectroscopy. A sensing layer of streptavidin immobilized inside the air holes of the PCF captures the antistreptavidin through an antigen-antibody interaction.

The selectivity of the capture has previously been documented using epifluorescence microscopy [2], but with the implementation of a highly sensitive setup [3], we are capable of measuring the $\mathrm{Cy} 3$ fluorescence spectrum, thereby giving a more sensitive and reliable detection. The PCF is illuminated from the side with a line-shaped laser beam. The fluorescence emitted from the $\mathrm{Cy} 3$ molecule tunnels through the evanescent field into the fiber cores and is guided to the fiber end.

\section{MOTIVATION}

The air holes of a photonic crystal fiber allow the positioning of liquid and gaseous samples in close proximity to the fiber core without removing the cladding of the fiber. Robust evanescent-wave absorption [4] and fluorescence sensors with a strong interaction between light and sample can thus be realized. The detection of antibodies produced in the human body may be used as diagnostic targets of diseases.

\section{STRONG-PENETRATION PHOTONIC CRYSTAL FIBERS}

Photonic crystal fibers form a recent class of optical fibers based on a photonic crystal pattern of air-holes running along the length of a silica fiber.
Careful choice of the physical characteristics is needed to obtain a strong penetration depth of the optical field inside the air holes of a PCF. Usual index-guiding PCFs with a solid core and a lattice of surrounding air holes only provide interaction between light and a small fraction of the sample volume, due to the relatively high confinement of the light in the core of the fiber. Light guidance inside a hollow core through the photonic bandgap effect provides maximal interaction between the guided light and the deposited sample. But these fibers are still difficult to design and fabricate. Another possibility is to use a hollow core PCF and to guide the light inside the air-suspended silica structures of the cladding, acting as a high number of cores. The size of these silica cores $(\approx 1 \mu \mathrm{m}$ wide $)$ and their proximity to the surrounding air holes realize better fluorescence signal conveyers than more traditional PCF approaches. This type of PCFs is referred to as Strong Penetration Photonic Crystal Fibers (SP-PCF).

The SP-PCF chosen for these experiments is provided by Crystal Fibre A/S and has a microstructured part composed of a large hollow core $(17 \mu \mathrm{m}$ in diameter) and a triangular lattice (pitch $4 \mu \mathrm{m}$ ) of smaller holes presenting different diameters $(4.2 \mu \mathrm{m}$ and $3.4 \mu \mathrm{m})$, as shown in figure 1 .

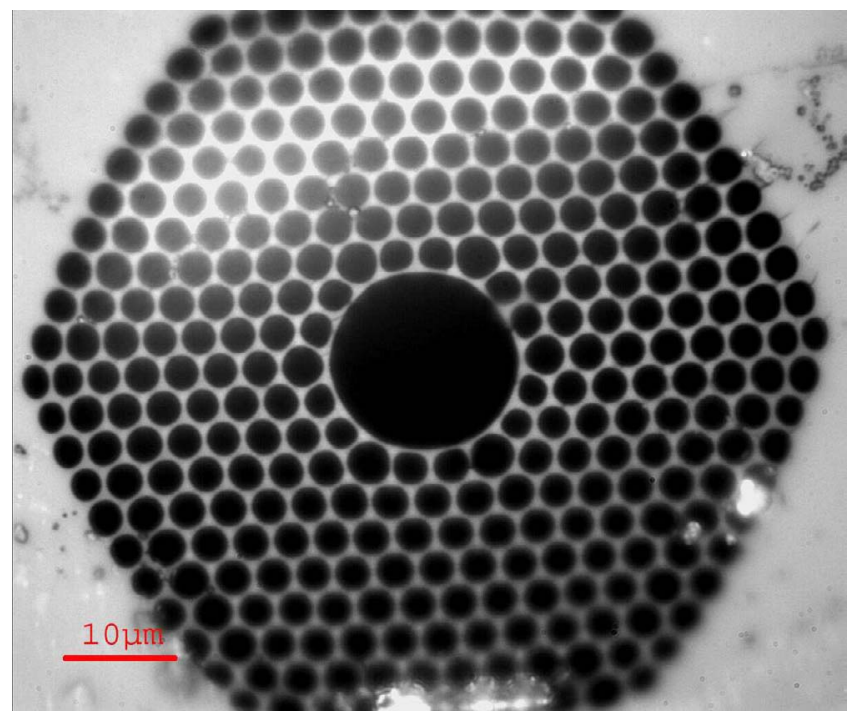

Figure 1. Optical micrograph of the cleaved end-facet of a SP-PCF, only the microstructured part is shown. 
Table 1. Coating procedure prior to protein binding

\begin{tabular}{|c|c|c|}
\hline Step & Solution & Duration \\
\hline 1 & Poly-L-Lysine $\left(1: 100 \mathrm{in}_{2} 0\right)$ & $15 \mathrm{~min}$ \\
\hline 2 & Glutardialdehyde $(12.5 \%)$ & $45 \mathrm{~min}$ \\
\hline 3 & Streptavidin in PBS $\left(1 \mathrm{mg} \cdot \mathrm{mL}^{-1}\right)$ & $30 \mathrm{~min}$ \\
\hline 4 & Ethanolamine $(40 \mathrm{mmol})$ & $20 \mathrm{~min}$ \\
\hline
\end{tabular}

\section{ANTIBODY IMMOBILIZATION}

Prior to immobilizing the proteins in the air-holes of the SPPCF, a biochemical coating is deposited to ensure trapping of the biomolecules at the glass/air interface. The air-holes are kept under a pressure difference of $200 \mathrm{kPa}$ in order to shorten the filling times. The complete chemical coating procedure is detailed in table 1 .

Between each step of the coating procedure, the air-holes are filled with a $\mathrm{pH}$ neutral solution of Phosphate Buffered Saline (PBS) at $10 \mathrm{mmol}$ during $5 \mathrm{~min}$ to stop the chemical reactions. The first layer deposited at the glass interface is an aqueous solution of poly-L-lysine acting as a positivelycharged substrate, covered then by a negatively charged layer of glutardialdehyde which will bind to the poly-Llysine. The streptavidin sample is then injected in the fiber and binds to glutardialdehyde sites by electrical affinity. Finally, the empty sites of glutardialdehyde are blocked by positively-charged ethanolamine molecules. A schematic representation of the layers arrangement is given in figure 2.

The selective immobilization of antibodies in the SP-PCF based sensor is ensured by filling the coated fiber with either a solution of $0.05 \mathrm{mg} . \mathrm{L}^{-1}$ unspecific alpha-CRP-Cy3 oligoprotein (unspecific) or a solution of $0.05 \mathrm{mg} . \mathrm{L}^{-1}$ antistreptavidin-Cy3 oligoprotein (specific binding) during $1 \mathrm{~h}$. A subsequent PBS wash ensures that only the bound antigen- antibody pairs remain present inside the air-holes of the SPPCF. A simplified representation of the specific and unspecific binding processes is given in figure 3 .

The presence of $\mathrm{Cy} 3$ fluorophores attached to the oligoprotein targets enables an evanescent fluorescent signal to be transmitted via the silica to the spectrometer when excited by a laser.
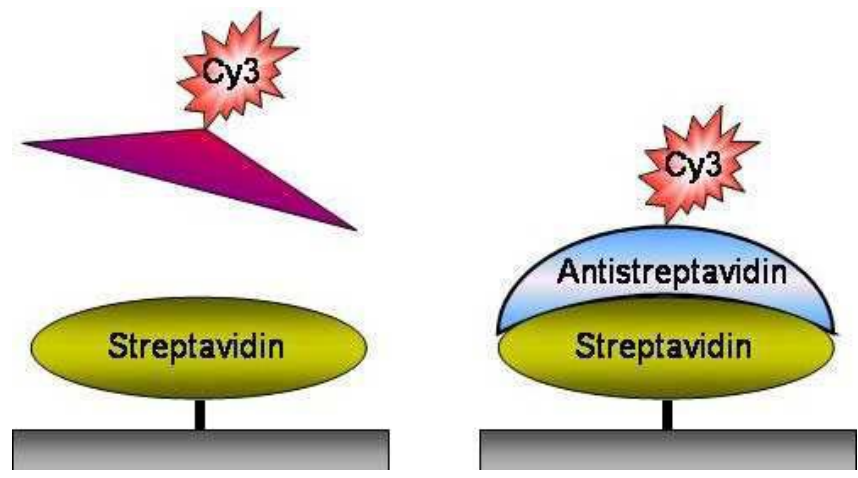

Figure 3. Representation of unspecific (left) and specific (right) streptavidin-antistreptavidin bounds inside the SP-PCF sensor.

\section{EXPERIMENTAL SETUP}

Samples of $30 \mathrm{~cm}$ of SP-PCFs were prepared as described in the previous section and a fluorescence measurement was performed with both an unspecific and a specific sample.

To enable strong penetration of the laser light inside the fiber, we stripped the acrylate coating of a $7 \mathrm{~cm}$-long section of SP-PCF and suspended it between two Z-alignment stages. A $20 \mathrm{~mW} \mathrm{CW}$ line-shaped laser (aperture $45^{\circ}$ ), emitting at $532 \mathrm{~nm}$ was used to illuminate the fiber from the side. Perfect alignment between the laser and the fiber is realized by observing the resulting diffraction patterns.

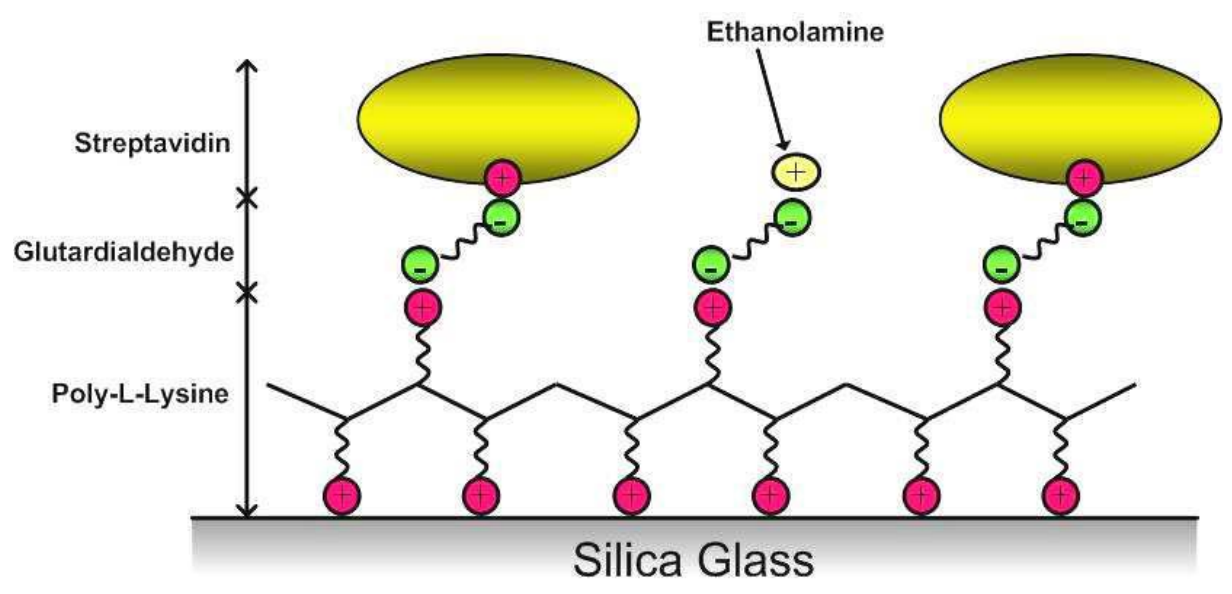

Figure 2. Coating of the SP-PCF. The electrical affinities between the different molecules are shown. 


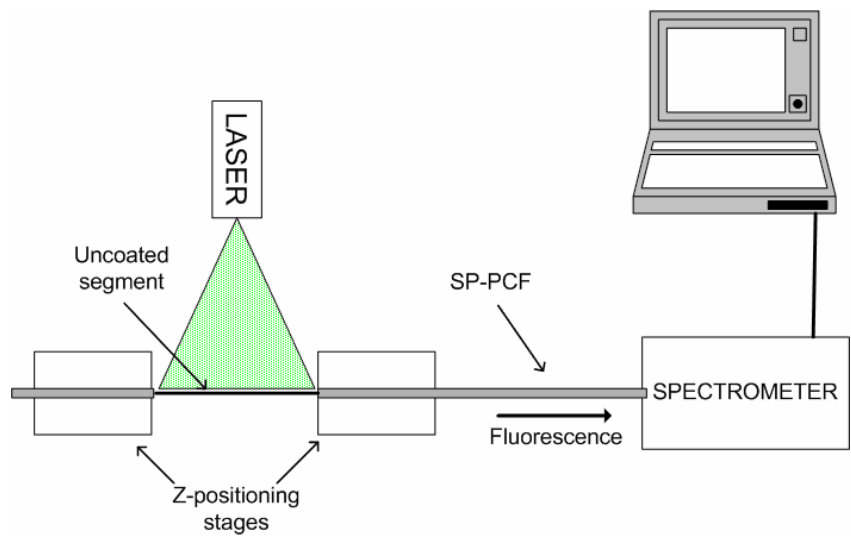

Figure 4. Block diagram of the setup. The line-shaped laser beam illuminates the fiber from the side with a highly efficient excitation of the Cy3 labeled molecules. The fluorescent signal is transmitted through the fiber and the transmission spectrum is analyzed with a spectrometer.

The choice of a line-shaped laser is motivated by the ease of use of this method and its fast alignment procedure. Indeed, no coupling has to be realized between the light and the fiber. The wavelength emitted by the laser matches the excitation peak of the $\mathrm{Cy} 3$ fluorophore present in the target oligoprotein molecules, which in turn emit at a slightly longer wavelength (peaking at $620 \mathrm{~nm}$ ).

The light transmitted though the fiber is detected by the spectrometer and the fluorescence signal from the $\mathrm{Cy} 3$ fluorophores can be observed as a transmission peak in the $620 \mathrm{~nm}$ region. A simplified representation of the experimental setup is depicted in figure 4.

We chose for this experiment a high precision Ocean Optics HR2000 spectrometer, detecting in the visible spectrum $(\approx 400-800 \mathrm{~nm})$ with a $1 \mathrm{~nm}$ resolution. The integration time is fixed at $100 \mathrm{~ms}$ in our experiment, since the concentration of fluorophores is large enough to saturate the spectrometer for longer durations.

It must be noted that no fluorescence decay of $\mathrm{Cy} 3$ fluorophores was observed, and thus no particular precautions were taken towards controlling the fiber exposure time to the laser light.

A picture of the actual setup is shown in figure 5 .

\section{RESULTS AND DISCUSSION}

We placed 4 chemically coated SP-PCFs in the pressure chamber for antibody target filling during $1 \mathrm{~h}$. Two fibers were filled with a solution of unspecific alpha-CRP-Cy3 oligoprotein, the two others with a solution of specific antistreptavidin-Cy3. After filling, the air-holes were washed during $6 \mathrm{~min}$ with PBS. The results of two representative samples are shown in figure 6.

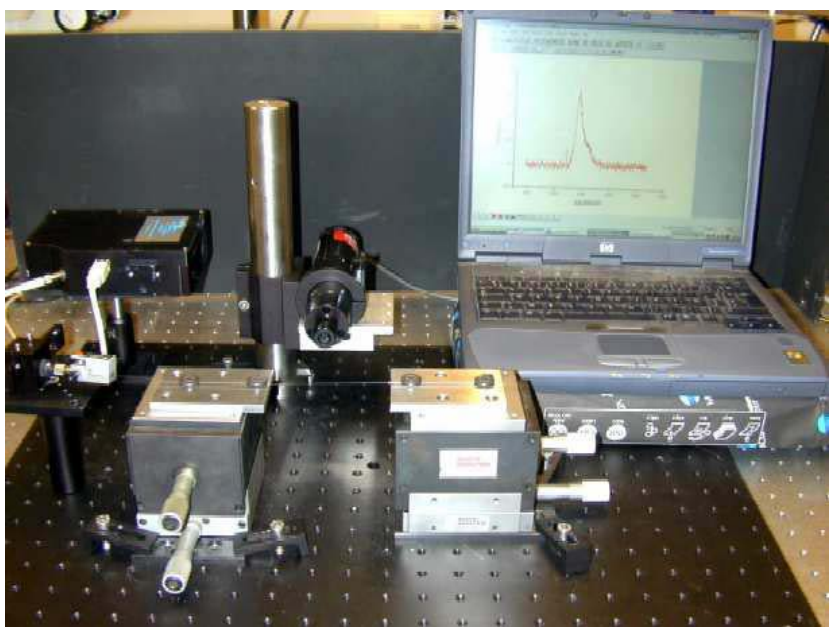

Figure 5. Picture of the experimental setup. Two Zstages are used to position the fiber relative to the line-shaped laser beam, and the spectrometer can be seen on the left of the setup.

These results show that the selective sample exhibits a stronger fluorescence than the non-selective sample (fluorescence approximately twice as intense). Re-absorption of the fluorescent signal by the fluorophores present in the fiber is observed and explains the lack of fluorescence in the $580 \mathrm{~nm}$ region. The selective detection was confirmed by an epifluorescence microscopy measurement.

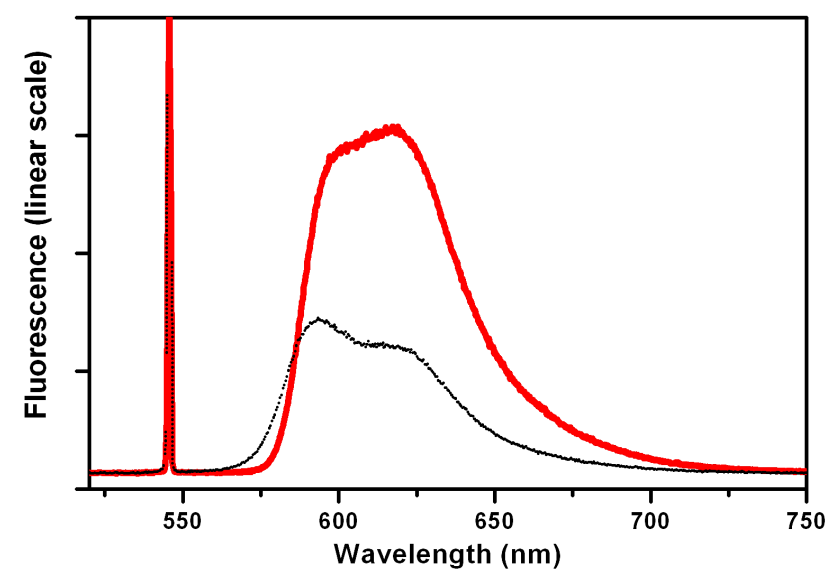

Figure 6. Fluorescence spectrum detected by the spectrometer. (thick curve) specific sample, (thin curve) unspecific sample.

When working with PCFs for biosensing applications, one should be aware of problems arising due to the size of the microstructures. The flow of liquids is governed by the laws of microfluidics and the surface tension is predominant in such channels. Liquids are thus more difficult to evacuate completely and residual unbound $\mathrm{Cy} 3$ fluorophores are present even after long washing times. Furthermore, a differential filling rate within the fiber has been observed, leading to non-homogeneities in the coating. 
The final PBS filling duration after binding is also an issue, since $6 \mathrm{~min}$ is obviously too short and leads to a strong fluorescence level from the unspecific sample.

Using the same unspecific sample, a subsequent PBS wash of $10 \mathrm{~min}$ was done and the fluorescence level was measured. Another measurement was realized after flushing the same fiber under a pressure difference of $200 \mathrm{kPa}$ for $10 \mathrm{~min}$. The results are shown in figure 7.

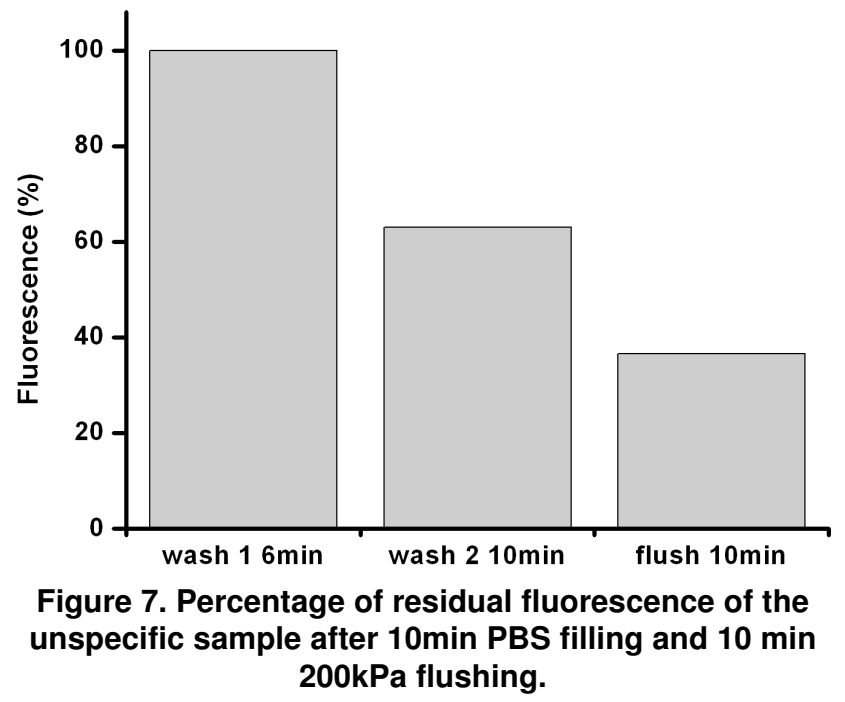

These results clearly show that the residual fluorescence coming from the remaining liquid solution of alpha-CRPCy3 molecules becomes negligible after longer washing and flushing periods. This yields the need for a more precise mean of determining the filling dynamics of SP-PCFs to be able to reliably detect specific antibodies binding.

\section{CONCLUSIONS}

We demonstrated here an original approach for detecting antibodies based on the properties of strong penetration photonic crystal fibers. Selective sensing of antibodies has been realized. The overall reliability can be improved by tuning the filling times to match the size of the geometry in consideration (microns-wide flow channels). The improvement of this method will be subject to further work.

\section{ACKNOWLEDGMENTS}

This work was supported by a grant to Anders Bjarklev from the Technical Council of Denmark and by a grant to the Ministry of Science, Technology and Development provided to the Center for Optical Sensors (COS).

\section{REFERENCES}

[1] J.C. Knight, T.A Birks, P.St.J. Russell, D.M. Atkin, "All-silica Single-mode Optical Fiber with Photonic Crystal Cladding”, Opt. Lett. Vol 21, pp. 1547-1549, Oct. 1996

[2] P.E. Hoiby et al., "Molecular Immobilization and Detection in a Photonic Crystal Fiber", BIOP2004, Photonics West 2004, January 2004, San Jose, CA.

[3] J.B. Jensen et al., "Selective Detection of Labeled DNA Using an Air-clad Photonic Crystal Fiber", CLEO2004, May 2004, San Francisco, CA.

[4] J.B. Jensen, L.H. Pedersen, P.E. Hoiby, L.B. Nielsen, T.P. Hansen, J.R. Folkenberg, J. Riishede, D. Noordegraaf, K. Nielsen, A. Carlsen, A. Bjarklev, "Photonic Crystal Fiber Based Evanescent-wave Sensor for Detection of Biomolecules in Aqueous Solutions", Opt. Lett. Vol. 29, pp. 1974-1976, Sept. 2004 